\title{
A Case of Meningitis? What's Your Diagnosis?
}

\author{
Pollach Gregor ${ }^{1}$, Kampondeni Sam ${ }^{2}$ \\ ${ }^{1}$ Department of Anaesthesia and Intensive Care, College of Medicine, University of Malawi, Blantyre, Malawi \\ ${ }^{2}$ Department of Radiology, College of Medicine, University of Malawi, Blantyre, Malawi \\ Email: gipi.bc62@yahoo.de
}

Received July 25, 2012; revised August 28, 2012; accepted September 16, 2012

\begin{abstract}
The five year old, somnolent girl from Mozambique with no medical history was presented with fever, neckstiffness, headaches, an almost complete paraplegia and a septic picture. Malaria parasites and HIV testing was negative. The lumbal puncture revealed staphylococcus. Subsequently a MRI scan showed no sign of meningitis but an extensive posterior epidural collection from C3 to the level of S1. Moreover it showed a vertebral osteomyelitis at L1. Bacteria in CSF were seen as an artefact and the child was treated by multilevel (purely) epidural punctures with drainage, rinsing and instillation of ceftriaxon. The epidural puncture proved acid fast bacilli in the pus, so that an anti-tuberculotic therapy was given. The child recovered quickly from sepsis and was able to walk several steps without help after three months.
\end{abstract}

Keywords: Meningitis; MRI; Mozambique; Epidural Puncture; Paraplegia

\section{Clinical History}

A five-year-old, somnolent girl from a rural region in Mozambique was brought to our hospital in Malawi by her mother. She presented with fever, tachypnoea, tachycardia, a relatively low blood pressure (which was difficult to measure due to a lack of correctly sized pressure cuffs), neckstiffness, headaches and intense back pain at the level L1). On physical examination the child showed an almost complete paraplegia of the inferior limbs with loss of sensation and incontinence.

The girl had no medical history and her mother did not remember a trauma, an accident with a dog, a snakebite or any form of traditional treatment.

A full blood count demonstrated leucocytosis and anaemia. Malaria parasites and HIV testing were negative

A lumbal puncture was initiated. Our setting in a resource poor country makes it impossible to do a CT or an MRI before the LP in order to exclude a raised intracranial pressure. The LP showed a turbid cerebro spinal fluid. A turbid spinal fluid usually is due to a mixture of bacterial and cellular debris with a huge amount of leucocytes. This turbid CSF than is very much an indicator of an acute bacterial meningitis. The culture of the CSF re- vealed Staphylococcus aureus.

Leucocytosis, tachycardia, tachypnoe and a reduced GCS, together with fever and a borderline bloodpressure constitute the diagnosis of a systemic inflammatory response syndrome in our patient. The additional finding of Staphylococcus aureus in the pus as source for the SIRS defines the diagnosis of an SIRS through infection, commonly called sepsis.

Our clinical problem now was, that a paraplegia with sensory loss and incontinence did not fit to the diagnosis of meningitis. In meningitis we should expect a hemiplegic picture. Due to this problem we decided to make use of our scarce resources and initiated a MRI scan.

\section{Imaging}

A MRI of the vertebral column showed no signs of meningitis but an extensive posterior epidural collection of fluid from C3 to the level of S1 with two suspicious structures, appearing like abscesses on the level Th12/L1 (Figures 1 and 2).

Figure 1 shows a sagittal, T2-weighted MRI, where there is a high-intensity signal parallel to the spinal cord. Proofed by aspiration this was an epidural collection of pus from the cervical to the sacral spinal cord (exactly: C3 to S1). There were additional pockets of pus on two levels (Th12/L1).

Figure 2 shows us a coronal T2-weighted MRI on the level of L1 with a collection of fluid with a high-intensity signal posterior to the spinal channel and the spinal cord. There is no suspicious signaling in the spinal channel or around the spinal cord. The fluid was diagnosed as an epidural empyema.

Sepsis as well as the progressive respiratory impairment (due to an ascending compression of the spinal cord) became lifethreatening. The hospital (the central hospital 




Figure 1. Abscess or empyema-Sagittal.

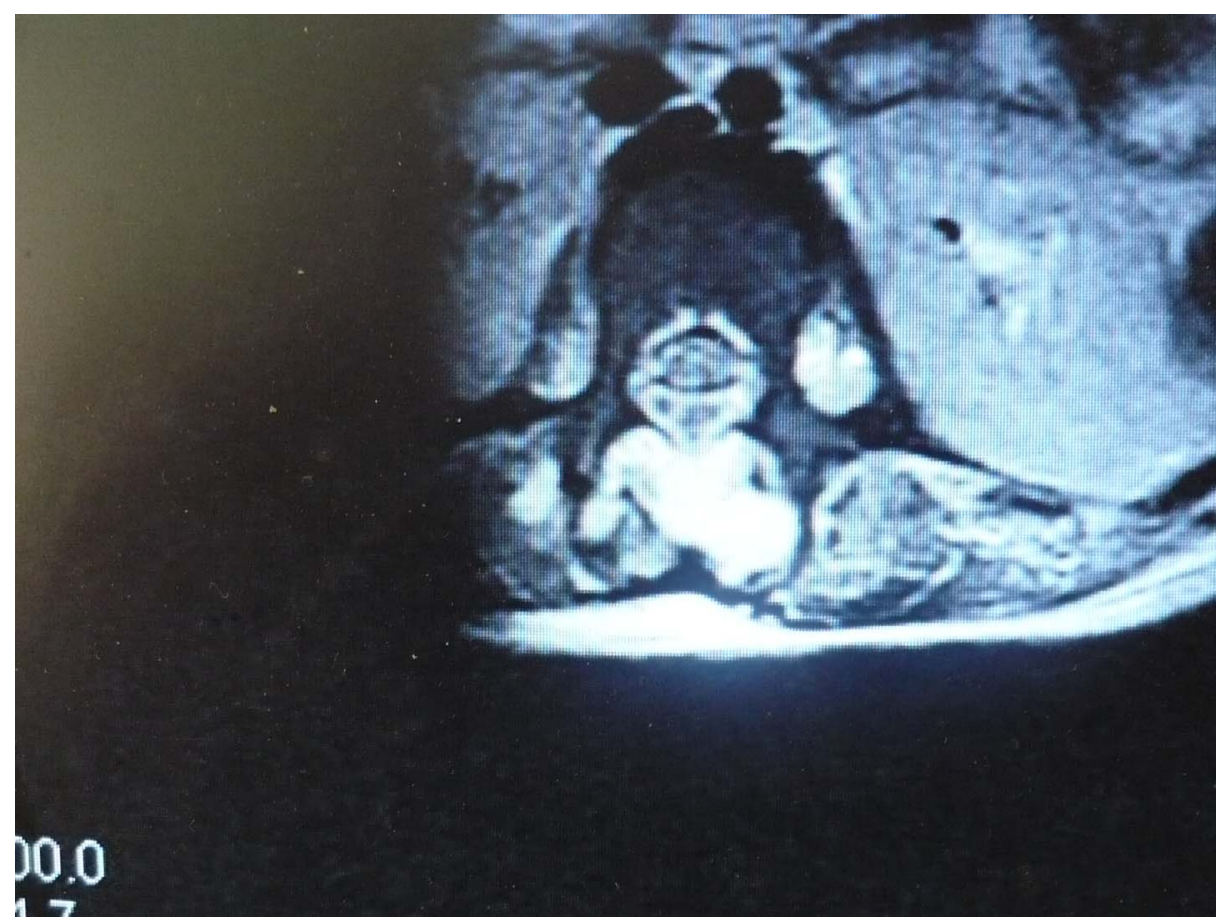

Figure 2. Abscess or empyema-Coronal. 
of the region) had no neurosurgeon or orthopaedic spine surgeon during this period. Without a conservative alternative $[1,2]$ the child was treated through anaesthesia by multilevel epidural punctures with the drainage of 40 $\mathrm{ml}$ pus. After the drainage we inserted temporary epidural catheters from TH7/8 to L3/4 for an extensive irrigation with sodium chloride $(0.9 \%)$ and an epidural instillation of ceftriaxon [3].

The febrile septic condition improved already during the next day and our little patient moved a little bit her right leg-but a second MRI (Figure 3) showed again an epidural fluid collection.

Figure 3 shows a sagittal T2-weighted MRI, where despite aspiration of around $40 \mathrm{ml}$ pus the day before we see a similar distribution of pus in the epidural space as in the first MRI (Figure 1). Moreover we saw on the level of L1 a high intensity signal from the marrow of the vertebral body of L1. The level of the enhanced signal was consistent with the level of the cranial pocket of the epidural empyema. In our tropical setting with its high prevalence of spinal tuberculosis this is very suspicious for a tuberculous osteomyelitis of the spine [4]. Pus related to a vertebral tuberculous osteomyelitis spreads locally and gives (again) a high-density signal in the T2 weighted MRI [4].

Following this second MRI we drained again $30 \mathrm{ml}$ of cloudy fluid from the epidural space. The fluid was clearly less thick this time (and the child was clinically better), so that we did not thought of a new collection of pus but of a redistribution and mixture of not drained pus with the instilled irrigation fluid. This time we were able to discover mycobacteria in the drained fluid.

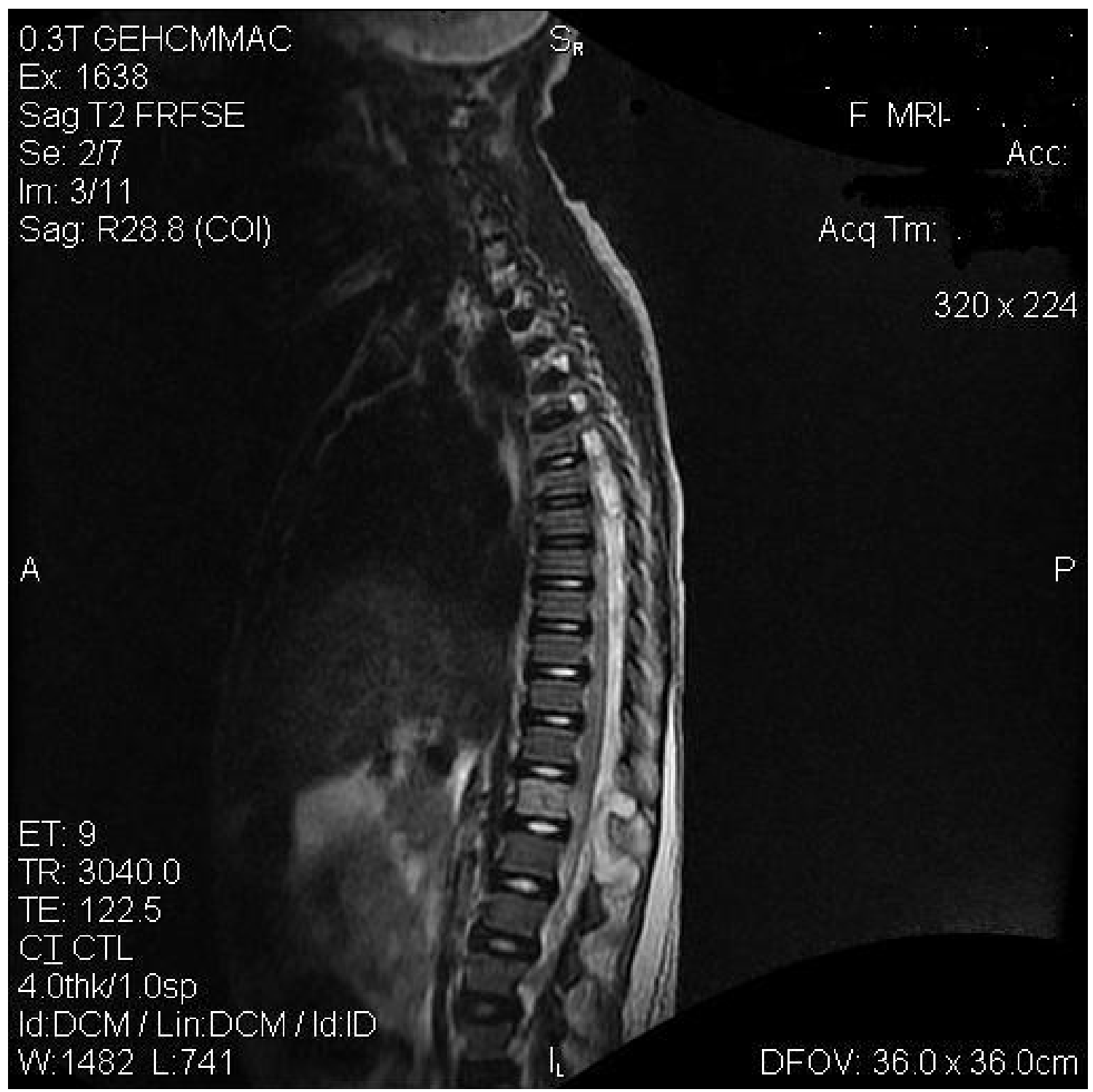

Figure 3. Empyema plus osteomyelitis. 


\section{Comment and Discussion}

After the second epidural intervention and an intravenous therapy with ceftriaxon, flucloxacillin, isoniazid, rifampicin, pyrazinamide and ethambutol we saw a rapid improvement of the clinical picture.

Prompt recovery from the systemic inflammatory response syndrome as much as the improvement of the paraplegia with the parallel loss of the local symptoms which were suspicious of meningitis (nuchal rigidity and headaches) followed a strictly epidural intervention. The changes we saw in the MRI (concerning only the epidural space and not the subarachnoidal space) pointed to the fact that we saw really an purely epidural problem without involvement of the subarachnoidal space. These were indicators, that the diagnosis of meningitis, despite a positive and turbid CSF, was wrong. We had to conclud that the infection never reached the subarachnoidal space and that there was no meningitis at all. Bacteria in the CSF had to be seen as an artefact through a needle which went either only into the enlarged epidural empyema but not into the subarachnoidal space or which were drawn from the epidural space into the subarachnoidal space-so that the puncture was erraneously seen as having produced CSF with pus.

For the colleague who performed the first LP it was probably not possible to avoid this contamination because he was not aware (the MRI was done later) of the epidural pathology. An epidural empyema is so uncom- mon that we cannot have expected him to think of condition. The finding of Staphylococcus aureus in the drawn fluid in a clinical situation with acute fever, headaches and nuchal rigidity is strongly indicative of an acute bacterial meningitis. In this situation there is no real differential diagnosis for the cloudy CSF, as long as you accept it as fluid from the subarachnoidal space.

Because paraparesis is uncommon in such a situation and you would much more expect a hemiparesis in meningitis we decided to do MRI imaging-despite the fact that MRI imaging is very expensive and puts constrains on our departmental funds in the resource poor setting we live in. Through the MRI we were forced to revise our initial diagnosis of meningitis and had to find the correct explanation for the cloudy" CSF-like fluid".

The MRI (Figure 3) showed a vertebral body on L1 which was highly suspicious of tuberculosis. Besides the MRI imaging this assumption is backed by the high prevalence of thid condition in Malawi and the identification of mycobacterium tuberculosis in the epidural pus after the second drainage. In spinal tuberculosis we very often see paraspinal abscesses. We have to assume that the paraspinal abscess got access to another structure-in our case to the epidural space at the level L1 where we see a pocket of the epidural space in the sagital images a and c. Than this fluid was superinfected by Staphylococcus aureus-leading to the septic symptoms. Access to other structures is well known in tuberculous paraspinal

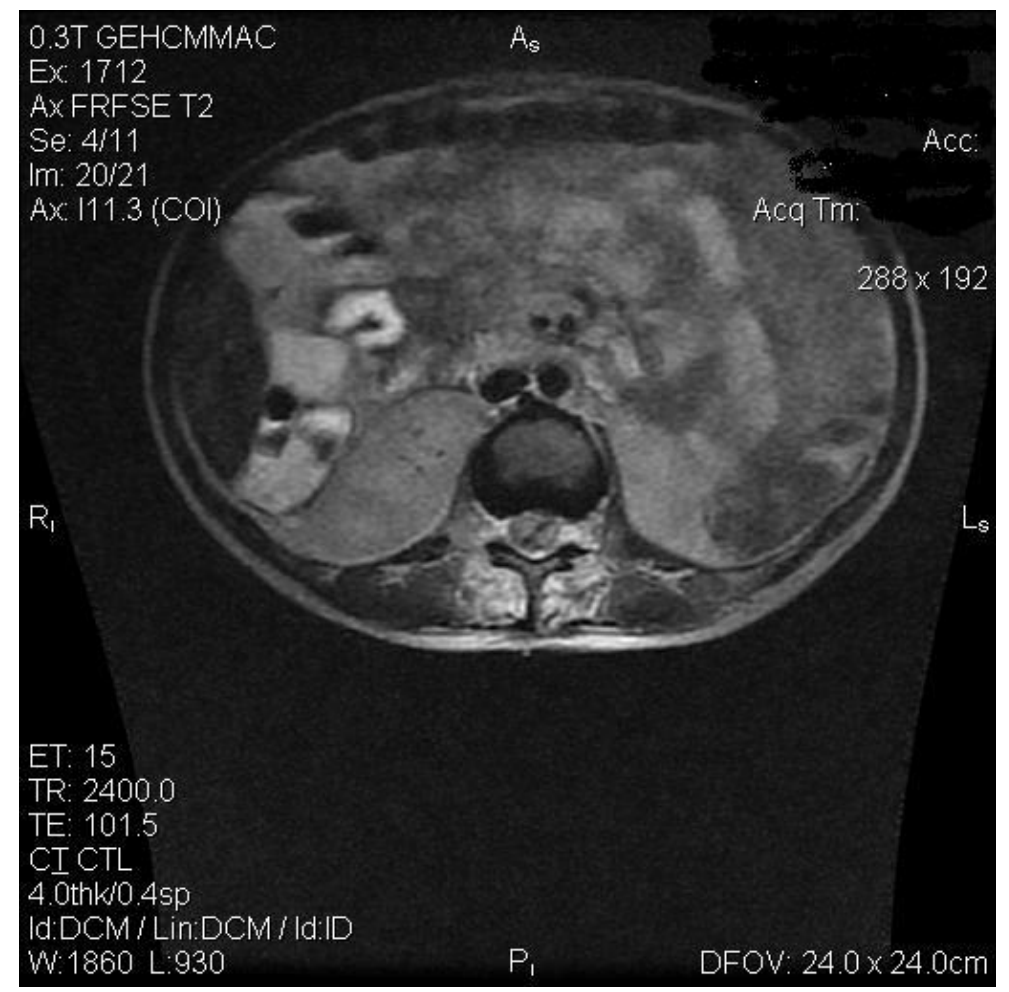

Figure 4. Resolution after therapy. 
abscesses (e.g. the connection towards the bowel through a psoas-abscess). The neurological symptoms in spinal tuberculosis than follow usually the pressure on the spine through the extraspinal abscess (or in this case through the extraspinal empyema) - and not through direct compression of a collapsing vertebral body. Paraplegia develops in more than $50 \%$ of the cases through this extravertebral compression - with a more or less intact and stabile vertebral body (as it can be seen in our MRIscans).

\section{Diagnosis}

Severe infectious systemic inflammatory response syndrome (severe sepsis) following a large spinal epidural empyema from C3 to S1 with paraplegia through compression of the spinal cord due to the empyema, following a tuberculous vertebral osteomyelitis.

\section{Outcome}

Our patient recovered quickly from sepsis and three months later an impressive improvement could be seen in the MRI (Figure 4: Sagittal T2-weighted MRI, which did not show (after the second aspiration of the epidural space) any enhanced signal in the epidural space. There is no suspicion of pus any longer in the epidural space, the CSF or anywhere in the whole subarachnoidal space. This correlates very nicely with the clinical picture.

Three months later the girl was continent, sensation was restored and she was able to walk some meters without help.

\section{REFERENCES}

[1] M. Gelabert-Gonzalez, J. Gonzalez-Garcia, J. M. Fernandez-Villa, A. GarciaAallut and R. Martinez-Rumbo, "Spinal Epidural Empyema. Analysis of 14 Cases,” Neurocirurgia (Astur), Vol. 15, No. 6, 2004, pp. 543-551.

[2] W. E. Krauss and P. C. McCormick, "Infections of the Dural Spaces," Neurosurgery Clinics of North America, Vol. 3, No. 2, 1992, pp. 421-433.

[3] A. A. Adeolu, A. O. Malomo and T. M. Shokunbi, "Intraoperative Irrigation with Ceftriaxone in Neurosurgical Patients,” The Internet Journal of Neurosurgery, Vol. 2, No. 2, 2005, p. 3.

[4] P. E. S. Palmer and M. M. Reeder, "The Imaging of Tropical Diseases,” British Journal of Radiology, Vol. 74, No. 883, 2001, pp. 475-494. 